\title{
Investigation on a Broken Solar Burst Type II during High Activities in AR1613 on 13th November 2012
}

\author{
Z. S. Hamidi ${ }^{1, *}$, N. N. M. Shariff ${ }^{2}$, C. Monstein ${ }^{3}$, W. N. A. Wan Zulkifli ${ }^{1}$, \\ M. B. Ibrahim ${ }^{1}$, N. S. Arifin ${ }^{1}$, N. A. Amran ${ }^{1}$ \\ ${ }^{1}$ School of Physics and Material Sciences, Faculty of Sciences, MARA University of Technology, \\ 40450, Shah Alam, Selangor, Malaysia \\ ${ }^{2}$ Academy of Contemporary Islamic Studies (ACIS), MARA University of Technology, \\ 40450, Shah Alam, Selangor, Malaysia \\ ${ }^{3}$ Institute of Astronomy, Wolfgang-Pauli-Strasse 27, Building HIT, Floor J, \\ $\mathrm{CH}-8093$ Zurich, Switzerland \\ *E-mail address: zetysh@salam.uitm.edu.my
}

\begin{abstract}
The present article is an attempt to analyze the solar burst Type II observations based on solar flare and Coronal Mass Ejections (CMEs) events. We choose an intriguing type II radio burst with a velocity of $1193 \mathrm{kms}^{-1}$ that occurred on 2012 November 13 at 2:04:20 UT. In this case, the study of solar radio burst type III is of paramount importance because of the fact that it helps to gain an insight of generation mechanisms of solar flare and Coronal Mass Ejections (CMEs) phenomena. Here, we have got a reasonably clear idea of the various forms under which the type III continuum emission may appear and potentially form a type II burst. However, in this case, the Type II solar burst only successfully forms a fundamental structure within the first few minute period, but broken suddenly before evolve a harmonic structure. This phenomenon is very interesting to be tackled and study. How the burst suddenly broken is still ongoing research seems the event is very rare and hard to be proved. There are a few questions that cause this unique situation which related to: (i) the intensity and duration of type III burst which also related to the classification of solar flare (ii) the probabilities CMEs to occur during that time and also the factor of the total amount of massive burst that exploded, Thus, we can conclude that the solar burst type III event still tells us an enigmatic characteristic from time to time due to the relationship of energetic particles and streams of particles with coronal magnetic fields and the pattern of Sun activity due to the 24th solar cycle. It might an interesting to study in detail the main factor that caused the Type II solar burst broken. Indirectly, it might because of the very intense of solar flares that make the percentage of energy of solar flare become more dominant rather than the acceleration of particles through the Coronal Mass Ejections. Thus, we realize that the potential energy during this event is higher than the kinetic energy of the particles.
\end{abstract}

Keywords: Sun; low frequency; solar radio; burst, type II; type III; e-CALLISTO 


\section{INTRODUCTION}

In radio astronomy, solar radio emission the observations of solar radio bursts by low spectral and spatial resolution instruments can provide the light curve and a crude spectrum of the whole flares and Coronal Mass Ejections (CMEs) which may consist of many distinct sources with different characteristics. It has also played an important role in monitoring the space weather sources. In major cases, the solar radio burst type III plays a fundamental role in solar burst studies [1]. This is true not only in the determination of solar radio burst type II, but other types as well.

In general, the formation of the Type II solar burst is due to electrons accelerated at an outward propagating coronal shock front [2]. One main significance of this type is that it is the earliest indicators of CME-driven shocks. The onset time of this type precludes the possibility of the CME driven shock causing it [3]. The motion of the shock through the radial plasma density profile can be observed based on the decreasing of the signal in frequency. One can deduce the propagation speed of the driving shock wave from eruption region. SRBT II were first identified by [4] and also discovered by [5] and classified as a broadband, lasting from 20 minutes to a few hours.

The theoretical basis for the plasma hypothesis of Type III solar burst was first introduced by [6] in the frequency range 500-10 MHz. Type III solar burst, a fast drift burst is the most common of the meter wavelength bursts. It can be considered as a pre-flare stage that could be a signature of electron acceleration [7]. It is well known that an isolated Type III solar burst can exhibit a wide range of forms [8]. During the near maximum cycle, the average rate of occurrence growing up to three detections per hour with duration of individual times about 10 seconds. This type is associated with solar flares and usually occurs before optical events. Further evidence showed that type III are generated in a weak-field region comes from the absence or low degree of circular polarization of the bursts. However, [9] against the theory and strongly agree that Type III [10] burst requires a very strong field to produce a fundamental and second harmonic of gyro frequency. The subject of nonlinear wave-wave interaction which involving interaction of electrostatic electron plasma that called as Langmuir waves active region radio emissions also have been studied [11-15] and the most recent and comprehensive ones can be found in a recent book dedicated to solar and space weather radiophysics [16].

\section{EXPERIMENTAL SETUP AND OBSERVATION}

A Log Periodic Dipole Antenna (LPDA) was mounted on the top of the rooftop of National Space Centre (ANGKASA) building at Sg. Lang, Banting, Selangor located at (N $\left.02^{\circ} 49.488^{\prime} \mathrm{E} 101^{\circ} 36.168^{\prime}\right)$ that covered the range of frequency from $85-470 \mathrm{MHz}$ [17]. This antenna is connected to the CALLISTO spectrometer via cable RG 58 and the modification, calibration process and basic testing of the antenna has been done in order to improve the quality of the system $[18,19,20,21,22]$. A preamplifier also is used to maximize the gain of the signal. All the data are automatically saved in FIT files. On the other hand, due to constrain of the interference factors, the range of $150 \mathrm{MHz}$ till $350 \mathrm{MHz}$ had been chosen as selected data $[23,24]$. Since the CALLISTO returns an output of a standard FITS file that represents a frequency versus time diagram, it is difficult to determine the types of solar flare. Thus, it is necessary to process the image creating a specific diagram that gives a clear radio signature of flare or CMEs. 
There are several sites of solar Compound Astronomical Low-frequency, Low-cost Instrument for Spectroscopy Transportable Observatories (CALLISTO) spectrometer successfully detected this burst between 2:03:30-2:06:00 UT on 13th November 2012 such as KASI Korea and SSRT Switzerland. We also successfully detected this burst at the National Space Agency, Sg Lang Banting Selangor [25]. In this data, we focused from 220-380 MHz region seems this is the best range with a very minimum of Radio Frequency Interference (RFI) [26]. Based on the data, the burst is formed within four minutes. There is also a long fast-drift type Type III solar burst was recorded before the formation Type II solar burst. This Type III solar burst is ejecting intermittently before and after the formation of Type II solar burst [27-32].

\section{RESULTS AND ANALYSIS}

In this section, a comprehensive analysis of Type III solar burst observation of shortduration in association with eruptions on the Sun and appear like a radio burst type II is presented. This observation allows for the mechanisms of evolution type II solar burst and local environment of the burst to be characterized. Yet, the burst is quite unique and interesting to be tackled. The burst is classified as a Type III solar burst. However, a clear structure of fundamental Type II solar burst can be observed. This event occurred on 13th November 2012 from 2:00:03 UT till 2:00:06 UT. It peaked with M2.0 solar flare at 2.04 UT.

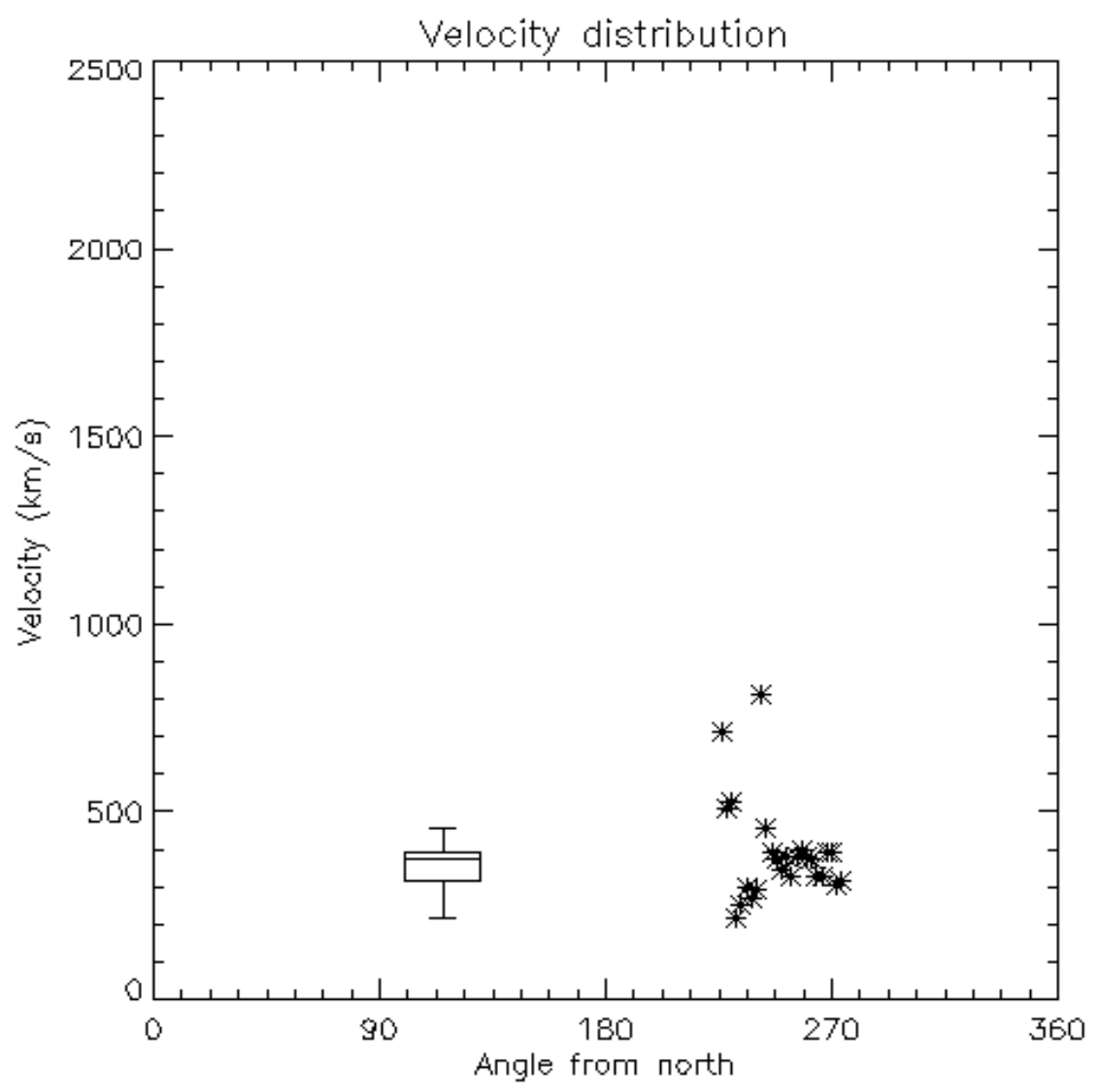

Figure 1. The velocity distribution of Coronal Mass Ejections at the first stage. Credited to: LASCO or SECCHI. 
In order to get a clear picture about this unique event, we also make use of full-disk imaging and coronagraph observations from the Solar Dynamics Observatory (SDO) satellite. This instrument has unprecedented high cadences and sensitivities with $0-1.5 \mathrm{R} \odot$ and 12 seconds. Figure 1 and 2 shows the velocity distribution of Coronal Mass Ejections at the first stage and during the explosion.

As can be seen in Figure 1, there is clearly a beginning of the evolution of CMEs explosion. The structure of CMEs tends to be formed. The velocity also starting to increase from $300 \mathrm{~km} \mathrm{sec}^{-1}$. It should be noted that this evolution occurred within a few minutes with a complex mechanism that not easy to be understood. This means that the tendencies of a huge explosion are very high and could be dangerous to the magnetic field of the Earth. This is a motivation for us to know more detail in understanding the mechanism of the explosion.

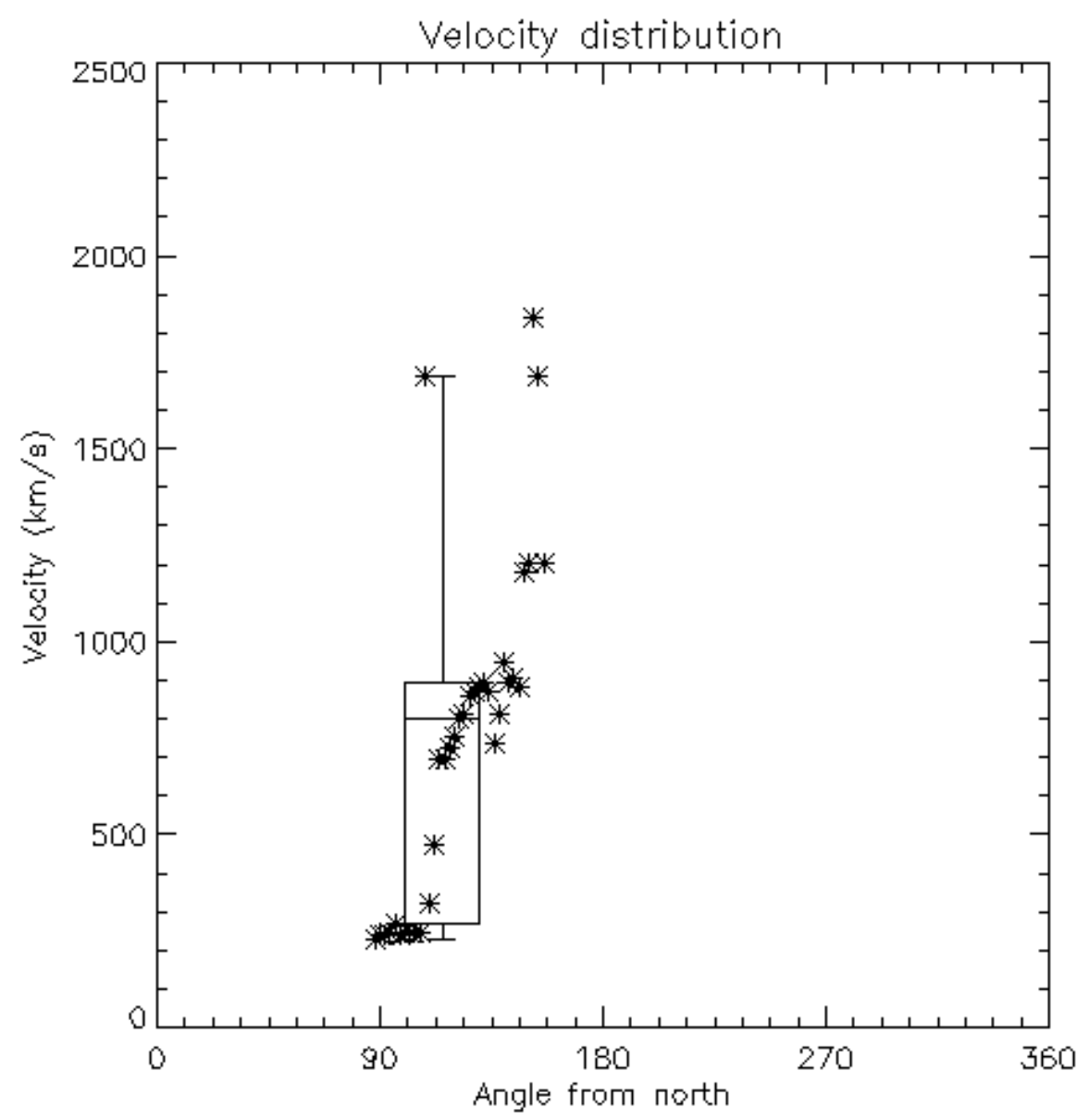

Figure 2. The velocity distribution of Coronal Mass Ejections at the during explosion. Credited to: LASCO or SECCHI.

Here, we used the data from LASCO2 to obtain a clearer picture on the range of distribution of the velocity of the CMEs. During that event, CMEs velocity also reached from 350-1900 $\mathrm{kmsec}^{-1}$. It shows a high acceleration of the particles from the thermal radiation. This parameter provided an alert with Sun's activity. The potential of a big explosion of CMEs during that period is considered very high. An explosion of CMEs by LASCO and SDO is illustrated in the next Figure. 


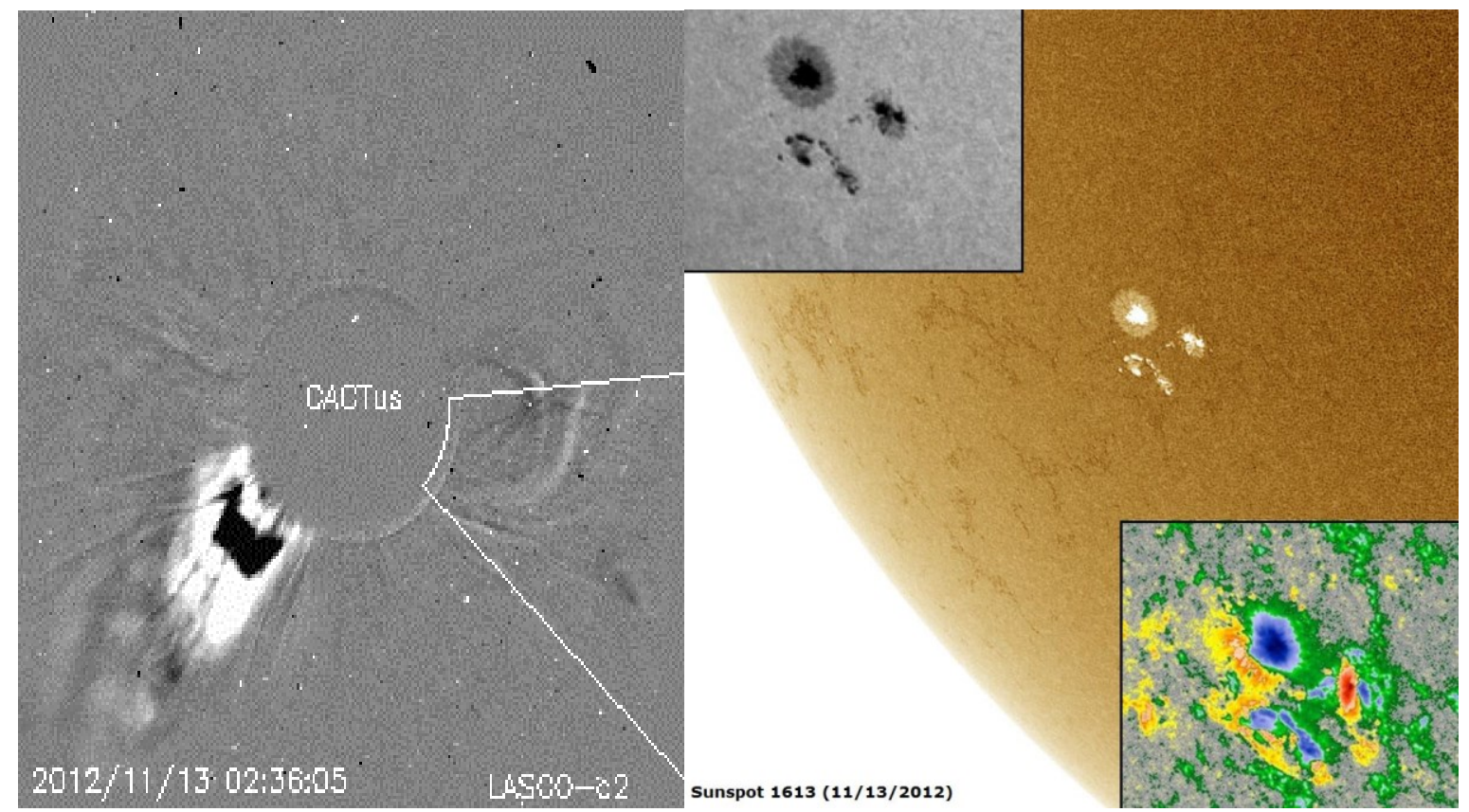

Figure 3. The significant explosion of Coronal Mass Ejections (CMEs) at 2:36:05 UT (left) and an Active Region 1613 (Credited to: Solar Dynamics Observatory (SDO) (right)).

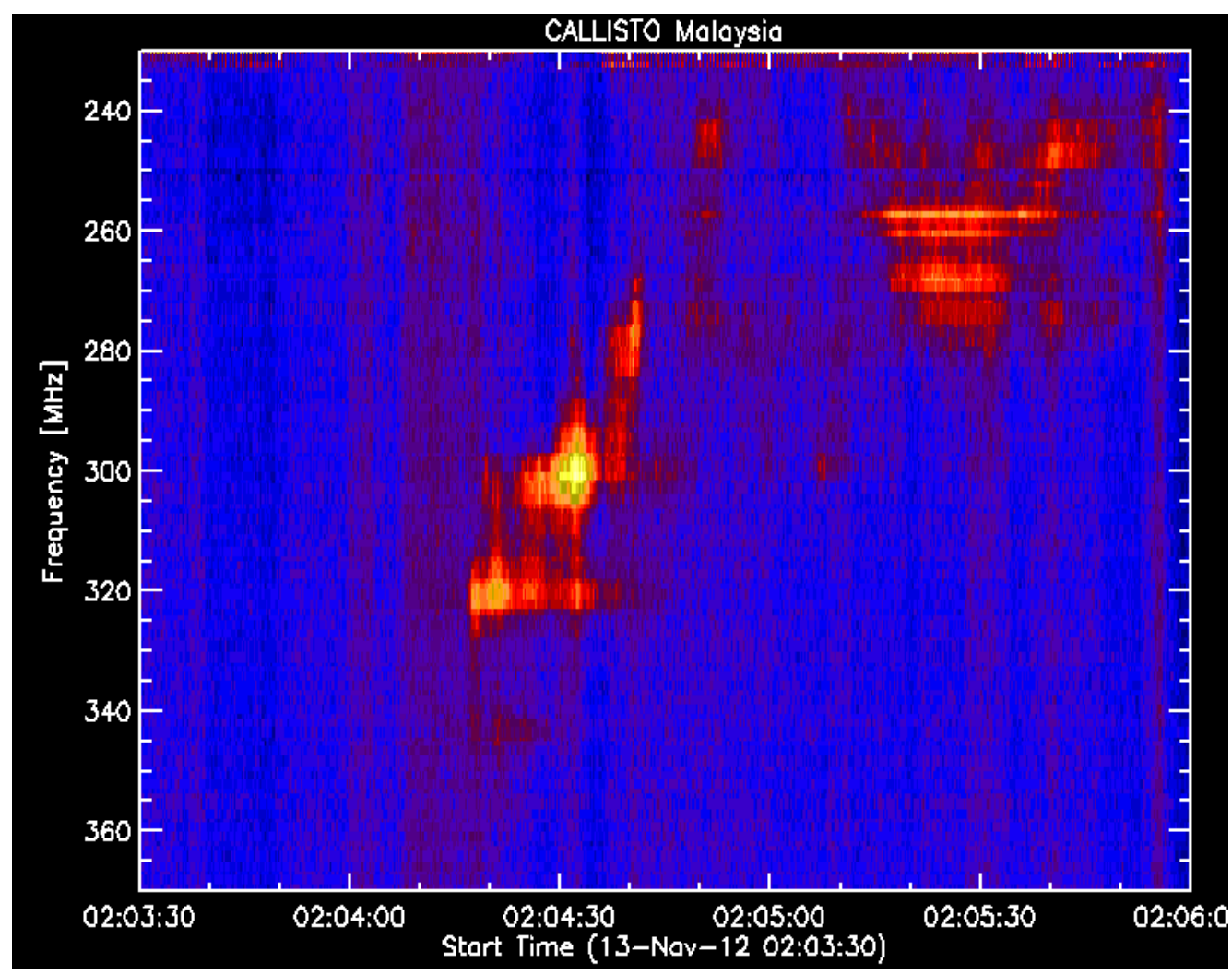

Figure 4. A broken solar burst type II with a fundametal structure. 
Figure 3 displays the explosion of CMEs by LASCO2 that occurred at 2:36:05 UT. The CMEs event is about 32 minutes after the formation of broken Type II burst. The range of radio dynamic spectrum recorded by CALLISTO system at the National Space Agency, Malaysia is from $220-380 \mathrm{MHz}$ and it was found that the Type II in the range of 240-320 $\mathrm{MHz}$.

From the observation, a fast drift Type III solar burst is formed intermittently within six hours period of observation starting fro 11:30 UT. Figure 4 shows the evolution of a broken Type II solar burst detected from our site.

We continue to analyze the next stage of this event. The burst that was temporally coincident with the X-ray region due to the M6.0 solar flare are are clearly seen. The type II burst started about two minutes later when the X-ray flux already declined to the background level. Before 2:04:15 UT, solar radio burst Type III burst can be identified. The type II burst is supposedly to be formed a harmonic structure. However, in this case, we could observe only a fundamental structure.

Overall, the Sun tends to be consistent with multiple storms, but not huge CMEs explosion. Based on LASCO observation, the CMEs explosion with a speed of $419 \mathrm{kms}^{-1}$ can be detected at 3:12 UT which means that almost after 1 hour and 10 minutes after the formation of this burst. In conclusion, this event is one fine example of tendencies SRBT III, which makes it possible to form SRBT II. The burst is form in between of two events of CMEs in at the same day.

\section{DISCUSSION AND CONCLUSION}

There must be a significant factor that caused the Type II solar burst broken. Indirectly, it might because of the very intense of solar flares that make the percentage of energy of solar flare become more dominant rather than the acceleration of particles through the Coronal Mass Ejections. Thus, we realize that the potential energy during this event is higher than the kinetic energy of the particles.

\section{ACKNOWLEDGEMENT}

We are grateful to LASCO,SDO/AIA, NOAA and SWPC make their data available online. This work was partially supported by the PPP UM PV071/2011B grants. Special thanks to C. Monstein from ETH Zurich, Switzerland who set up and gives us training on analyzing the data. Also to National Space Agency and National Space Centre for giving us a site to set up this project and support this project. Solar burst monitoring is a project of cooperation between the Institute of Astronomy, ETH Zurich, and FHNW Windisch, Switzerland, MARA University of Technology and University of Malaya. This paper also used NOAA Space Weather Prediction Centre (SWPC) for the sunspot, radio flux and solar flare data for comparison purpose. The research has made use of the National Space Centre Facility and a part of an initiative of the International Space Weather Initiative (ISWI) program.

\section{BIOGRAPHY}

Zety Sharizat Hamidi is currently a $\mathrm{PhD}$ candidate and study in Solar Astrophysics specifically in radio astrophysics at the University of Malaya. Involve a project under the International Space Weather Initiative (ISWI) and also a lecturer in School of Physics and Material Science, at MARA University of Technology, Shah Alam Selangor.

N.N.M..Shariff: Her current research is communicating sustainability. She is looking forward for cross-field research, i.e. solar astrophysics, light pollution measurement (mapping) and application of technology on sustainability. 
C.Monstein is a senior Engineer at Institute of Astronomy, Wolfgang-Pauli-Strasse 27, Building HIT, Floor J, CH-8093 Zurich, Switzerland and one of the researchers who initiated the CALLISTO system around the world.

W.N.A. Wan Zulkifli, is a final year student at School of Physics and Material Science, at MARA University of Technology, Shah Alam Selangor.

M.B. Ibrahim, is a final year student at School of Physics and Material Science, at MARA University of Technology, Shah Alam Selangor.

N.S. Arifin is a final year student at School of Physics and Material Science, at MARA University of Technology, Shah Alam Selangor.

N.A. Amran is a final year student at School of Physics and Material Science, at MARA University of Technology, Shah Alam Selangor.

\section{References}

[1] M. R. Kundu, Solar Radio Astronomy, John Wiley, 1965.

[2] N. Gopalswamy, P. Makela, H. Xie, S. A. Y. Akiyama, CME interactions with coronal holes and their interplanetary consequences, J. Geophys. Res. (2009a).

[3] N. Gopalswamy, N. Nitta, P. K. Manoharan, A. Raoult, M. Pick, Astronomy and Astrophysics 347 (1999) 684-695.

[4] R. Payne-Scott, D. E. Yabsley, J. G. Bolton, Nature 160 (1947) 256-257.

[5] A. Boischot, C. R. Acad. Sci. 244 (1957) 1326.

[6] J. P. Wild, Smerd S. F., Weiss A. A., Ann. Rev. Astron. Astrophysics 1 (1963) 291-366.

[7] G. A. Dulk, Type III solar radio bursts at long wavelengths, in: R. Stone, E. Weiler, M. Goldstein (Eds.), Geophys. Monogr., 2000.

[8] T. Takakura, Pub. Astron. Soc. Japan 12 (1960).

[9] A. F. Kuckes, R. N. Sudan, Sol. Phys. 17 (1971) 194.

[10] K. Kai, Sol. Phys. 11 (1970).

[11] G. B. Gelfreikh, Soviet Astron. 23 (1979).

[12] P. Lantos, Sol. Phys. 22 (1972).

[13] A. Vourlidas, Bastian T. S., Nitta N., Aschwanden M. J., Sol. Phys. 163 (1996).

[14] E. Y. Zlotnik, Soviet Astron. 12 (1968).

[15] V. V. Zheleznyakov, Radio Emission of the Sun and Planets (1970).

[16] D. E. K. Gary, C. U., Solar and Space Weather Radiophysics, Current Status and Future Developments Dordrecht: Kluwer, 2004.

[17] Z. S. Hamidi, Z. Ibrahim, Z. Abidin, M. Maulud, N. Radzin, N. Hamzan, N. Anim, N. Shariff, International Journal of Applied Physics and Mathematics 2 (2012) 3.

[18] Z. S.Hamidi, S. Chumiran, A. Mohamad, N. Shariff, Z. Ibrahim, N. Radzin, N. Hamzan, N. Anim, A. Alias, American Journal of Modern Physics 2 (2013) 4.

[19] Z. S.Hamidi, Z. Abidin, Z. Ibrahim, N. Shariff, C. Monstein, International Journal of Engineering Research and Development 3 (2012) 3. 
[20] Z. S.Hamidi, Z. Abidin, Z. Ibrahim, C. Monstein, N. Shariff, International Journal of Fundamental Physical Sciences 2 (2012) 32-34.

[21] Z. S.Hamidi, et al., International Journal of Fundamental Physical Sciences 2 (2012) 4.

[22] Z. S.Hamidi, N. N. M. Shariff, International Journal of Science and Mathematics 2 (2014) 3.

[23] Z. S. Hamidi, Z. Abidin, Z. Ibrahim, N. Shariff, Indication of radio frequency interference (RFI) sources for solar burst monitoring in Malaysia, ICPAP 2011, AIP Publisher, Indonesia, 2012, pp. 6.

[24] Z. S. Hamidi, N. N. M. Shariff, C. Monstein, The International Journal of Engineering 1 (2012) 3.

[25] Z. S. Hamidi, N. Shariff, Z. Abidin, Z. Ibrahim, C. Monstein, Middle-East Journal of Scientific Research 12 (2012) 6.

[26] Z. S. Hamidi, N. N. M. Shariff, R. Umar, Malaysia Thailand Journal of Physics 3 (2012) 6.

[27] [22] Z. S. Hamidi, N. N. M. Shariff, International Letters of Chemistry, Physics and Astronomy 5 (2014) 32-42.

[28] Z. S. Hamidi, N. N. M. Shariff, International Letters of Chemistry, Physics and Astronomy 5 (2014) 43-49.

[29] Z. S. Hamidi, N. N. M. Shariff, International Letters of Chemistry, Physics and Astronomy 7 (2014) 21-29.

[30] Z. S. Hamidi, N. N. M. Shariff, International Letters of Chemistry, Physics and Astronomy 7 (2014) 30-36.

[31] Z. S. Hamidi, N. N. M. Shariff, C. Monstein, Z. A. Ibrahim, International Letters of Chemistry, Physics and Astronomy 7 (2014) 37-44.

[32] Z. S. Hamidi, et.al., International Letters of Chemistry, Physics and Astronomy 8 (2014) 13-19. 